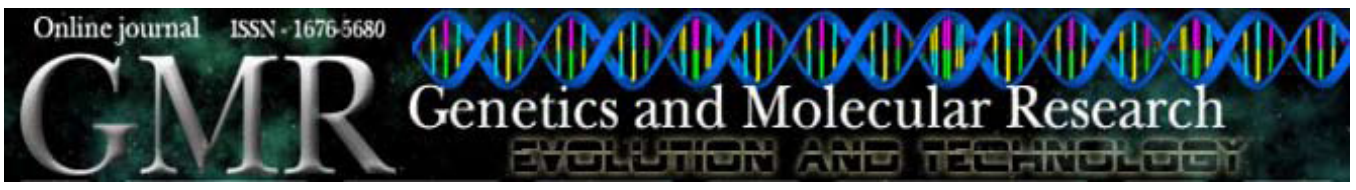

\title{
Precision of distances and ordering of microsatellite markers in consensus linkage maps of chromosomes 1, 3 and 4 from two reciprocal chicken populations using bootstrap sampling
}

\author{
M.F. Rosário1, G.R.A. Margarido ${ }^{1}$, C. Boschiero ${ }^{3}$, A.S.A.M.T. Moura ${ }^{3}$, \\ M.C. Ledur ${ }^{4}$, L.L. Coutinho ${ }^{2}$ and A.A.F. Garcia ${ }^{1}$ \\ ${ }^{1}$ Departamento de Genética, Escola Superior de Agricultura Luiz de Queiroz, \\ Universidade de São Paulo, Piracicaba, SP, Brasil \\ ${ }^{2}$ Departamento de Zootecnia, Escola Superior de Agricultura Luiz de Queiroz, \\ Universidade de São Paulo, Piracicaba, SP, Brasil \\ ${ }^{3}$ Departamento de Produção Animal, Faculdade de Medicina Veterinária e Zootecnia, \\ Universidade Estadual de São Paulo, Botucatu, SP, Brasil \\ ${ }^{4}$ Centro Nacional de Pesquisa em Suínos e Aves, EMBRAPA, Concórdia, SC, Brasil \\ Corresponding author: M.F. Rosário \\ E-mail: millor@usp.br
}

Genet. Mol. Res. 9 (3): 1357-1376 (2010)

Received March 20, 2010

Accepted May 18, 2010

Published July 13, 2010

DOI 10.4238/vol9-3gmr842

\begin{abstract}
Some factors complicate comparisons between linkage maps from different studies. This problem can be resolved if measures of precision, such as confidence intervals and frequency distributions, are associated with markers. We examined the precision of distances and ordering of microsatellite markers in the consensus linkage maps of chromosomes 1, 3 and 4 from two $F_{2}$ reciprocal Brazilian chicken populations, using bootstrap sampling. Single and consensus maps were constructed. The consensus map was compared with the International Consensus Linkage Map and with the whole genome sequence. Some
\end{abstract}


loci showed segregation distortion and missing data, but this did not affect the analyses negatively. Several inversions and position shifts were detected, based on $95 \%$ confidence intervals and frequency distributions of loci. Some discrepancies in distances between loci and in ordering were due to chance, whereas others could be attributed to other effects, including reciprocal crosses, sampling error of the founder animals from the two populations, $\mathrm{F}_{2}$ population structure, number of and distance between microsatellite markers, number of informative meioses, loci segregation patterns, and sex. In the Brazilian consensus $G G A 1$, locus $L E I 1038$ was in a position closer to the true genome sequence than in the International Consensus Map, whereas for GGA3 and $G G A 4$, no such differences were found. Extending these analyses to the remaining chromosomes should facilitate comparisons and the integration of several available genetic maps, allowing meta-analyses for map construction and quantitative trait loci (QTL) mapping. The precision of the estimates of QTL positions and their effects would be increased with such information.

Key words: Confidence interval; CRI-MAP; Linux; Seriation; Gallus gallus domesticus

\section{INTRODUCTION}

To date, three linkage maps have been constructed using molecular markers (RFLP, RAPD, AFLP, and SSR) from three reference chicken populations: Compton (Bumstead and Palyga, 1992), East Lansing (Levin et al., 1994) and Wageningen (Groenen et al., 1998). Groenen et al. (2000) integrated these three maps into a chicken consensus linkage map, covering $3800 \mathrm{cM}$, and Schmid et al. (2005) updated this map, covering $4200 \mathrm{cM}$. These maps have been used in many studies, including QTL mapping (Abasht et al., 2006), and bacterial artificial chromosome (BAC) and radiation hybrid physical mapping (Aerts et al., 2003; Ren et al., 2003; Wallis et al., 2004), in the assembly of the whole genome sequence of the chicken, which covers $1.25 \mathrm{~Gb}$ (Hillier et al., 2004). The genome sequence has been used to search for singlenucleotide polymorphisms (SNPs) that could be used for the construction of a more accurate and comprehensive physical map for the chicken (Wong et al., 2004; Wang et al., 2005). More recently, Groenen et al. (2009) published a new consensus linkage map, with the addition of 8599 SNPs, covering $3228 \mathrm{cM}$, using the East Lansing, Wageningen and Uppsala populations.

Several factors can influence the construction of linkage maps and their results. These include experimental population design (backcross or $\mathrm{F}_{2}$ ), genetic background and population size, number of molecular markers, number of phase-known informative meioses, segregation pattern of loci, genotyping errors, number of missing genotypes, and sex (Hackett and Broadfoot, 2003). These factors can make comparison between linkage maps from different studies difficult. Adopting measures of precision for estimates, such as confidence intervals for distances and frequency distributions for orders, based on the bootstrap sampling, might be a useful tool for measuring the uncertainty in each map and for comparisons.

In Brazil, the EMBRAPA Swine and Poultry Research Center and "Luiz de Queiroz" 
College of Agriculture/University of São Paulo (ESALQ/USP) developed two $\mathrm{F}_{2}$ reciprocal populations (TCTC and CTCT) from crossbreeding between broiler (TT) and layer (CC) lines. These lines have been adapted for tropical conditions of climate, nutrition, disease, and management and they have been used to develop commercial strains in Brazil (Figueiredo et al., 2003a,b).

Linkage maps were constructed for chromosomes 1 (Nones et al., 2005), 6, 7, 8, 11, and 13 (Ambo et al., 2008) for the TCTC population. Also, QTLs for growth and carcass traits were mapped on chromosome 1 (Nones et al., 2006) and genome scans for performance and fatness traits were carried out by Ambo et al. (2009) and Campos et al. (2009), respectively. The CTCT population was genotyped with markers from three chromosomes (GGA1, GGA3 and $G G A 4$ ), for which there was previous evidence for QTLs in the reciprocal cross (TCTC). This was done to narrowing down the search for QTLs. However, it would be useful to have joint linkage maps for these chromosomes, since they show evidence of QTLs. Combined linkage maps could provide better precision for estimates of marker distances and orders, improving QTL mapping.

Here, we report the precision of distances and orders of microsatellite markers on chromosomes 1, 3 and 4 consensus linkage maps from two Brazilian $\mathrm{F}_{2}$ reciprocal chicken populations, using bootstrap sampling.

\section{MATERIAL AND METHODS}

\section{Experimental populations}

The TCTC population was obtained from crosses between seven males from a broiler line (TT) with seven females from a layer line (CC) (TT x CC). The CTCT population was obtained in a similar way, using the reciprocal cross between the lines (CC x TT). In each population, these initial crosses produced the $\mathrm{F}_{1}$ generation (TC and CT), with seven full-sib families each. One male and three females were selected from each $\mathrm{F}_{1}$ family and each male was mated to three non-related $\mathrm{F}_{1}$ females. The resulting $\mathrm{F}_{2}$ generations (TCTC or CTCT) consisted of 21 full-sib families that produced approximately $100 \mathrm{~F}_{2}$ offspring each. Thus, about $4200 \mathrm{~F}_{2}$ offspring were obtained from both populations. Details are in Rosário et al. (2009).

Only the most informative $F_{1}$ were chosen for genotyping their $F_{2}$ progenies. This selection was based on two strategies: for TCTC, seven families for GGA1 (Nones et al., 2006) and six for GGA3 and GGA4 (Ambo et al., 2009) were selected, according to a selective genotyping step (Darvasi and Soller, 1992). Among these selected TCTC families, six were genotyped in at least two chromosomes. The numbers of $\mathrm{F}_{2}$ offspring genotyped were 648, 544 and 567 for GGA1, GGA3 and GGA4, respectively. For CTCT, a fixed number of four families was selected for the same chromosomes, according to the best combination of genotypic parameters in the $\mathrm{F}_{1}$ generation (number of genotypic classes, number of alleles and segregation patterns to be obtained in $\mathrm{F}_{2}$ ). A total of $356 \mathrm{~F}_{2}$ offspring were genotyped from the CTCT population.

\section{Genotyping}

DNA extraction, polymerase chain reactions (PCR) and genotyping steps were run as in Rosário et al. (2009). A total of 31 (13), 12 (12) and 7 (9) microsatellite markers were 
used in TCTC (CTCT) populations on GGA1, GGA3 and GGA4, respectively. For TCTC, markers were chosen according to Nones et al. (2006) and Ambo et al. (2009) and for CTCT, the selection of the markers was based on previous association with body weight at 42 days in the TCTC population. Primer sequences (forward and reverse) are available at ArkDB (http://www.thearkdb.org/).

\section{Error check}

For each population, $\mathrm{F}_{2}$ genotypes were compared among parents and grandparents to detect possible genotyping errors. The PEDCHECK program (Fishelson and Geiger, 2002; http://bioinfo.cs.technion.ac.il/superlink-online/makeped/pedcheck.shtml) was used for this purpose.

The number of double and triple recombinations was obtained with the CHROMPIC function using the CRI-MAP software (Green et al., 1990; http://linkage.rockefeller.edu/soft/ crimap/). When putative errors were detected, genotypes were checked, and corrected if necessary. These genotypes were used to obtain the number of phase-known informative meioses a posteriori. The segregation pattern for each locus in each family and population was analyzed using the chi-square test and classified according to four types: A (1:1:1:1), B (1:2:1), C (1:1), and D (no segregation). Bonferroni's correction was employed for joint control of type I error $(\alpha=0.05)$.

\section{Linkage map construction}

First, single maps were constructed for each population. Later, joint maps were also obtained, combining the genotype datasets from both populations, resulting in total sample sizes of 1004, 900 and $917 \mathrm{~F}_{2}$ offspring, for GGA1, GGA3 and GGA4, respectively. The procedures used for map construction were the same in both cases. Linear locus orders were obtained using the rank of loci, in agreement with the number of phase-known informative meioses a posteriori. The most likely order was determined based on comparisons of the likelihood of different orders. Distances between loci were estimated using multipoint estimates of recombination fractions, which were converted into map distances using the Kosambi's (1944) map function. The following options were employed in the analyses in the CRI-MAP software: TWOPOINT, BUILD, ALL, FLIPS2, and CHROMPIC, with LOD $=3$. These procedures resulted in an averaged map for both sexes. The first locus on each chromosome had its position based on the International chicken consensus linkage map (Schmid et al., 2005). Linkage maps were graphically presented using the MapChart software (Voorrips, 2002; http://www.biometris.wur.nl/uk/Software/MapChart/).

\section{Precision of distances and orders}

The procedures were based on the bootstrap method (Efron and Tibshirani, 1993) only for the Brazilian consensus linkage maps. For loci distances, these procedures were run in three steps: i) Using the segregating population, 1000 independent random samples with replacement were generated (bootstrap samples), all of the same size as the original data. These samples were obtained with individual reallocation, following what was suggested by Efron 
and Tibshirani (1993). This step was implemented using the R software (http://www.r-project. org/); ii) For each bootstrap sample, a linkage map was constructed for each chromosome, using the FIXED function of the CRI-MAP software, assuming that the order among the microsatellite loci was known, according to the results obtained from linkage map construction. Scripts were produced in GNU/LINUX SHELL language to process the analysis of each bootstrap sample, using the CRI-MAP software in LINUX with LOD $=3$. These maps were stored in a 1000-column matrix. Later, bootstrap estimates of the recombination fractions were used to obtain empirical distributions of these estimates; iii) Given the empirical distributions, confidence intervals were obtained with the percentile method (Liu, 1998). For each distribution, 2.5 and 97.5 percentiles were selected in order to achieve a 95\% confidence interval for each distance in the map.

A similar procedure was employed for locus order. Bootstrap samples were generated for each linkage group, and pair-wise recombination fraction estimates were obtained using the TWOPOINT function in CRI-MAP, with $\mathrm{LOD}=0$. Next, markers were ordered using the seriation algorithm (Buetow and Chakravarti, 1987), which could provide more reliable estimates of marker order, according to Mollinari et al. (2009). Each bootstrap order estimate was stored in a matrix, where lines corresponded to markers and columns corresponded to positions on the linkage map. Locus frequency distributions were plotted. If the estimated order for a linkage group is reliable, the diagonal elements of this matrix will show large frequencies, concentrating points on the diagonal (Liu, 1998).

\section{RESULTS}

\section{Brazilian single and consensus linkage maps}

\section{GGA1}

For the TCTC and CTCT populations, on average, 568 and $342 \mathrm{~F}_{2}$ individuals were genotyped, respectively, showing 3.4 and 3.5 alleles per locus and 596 and 353 phase-known informative meioses a posteriori. In TCTC, $60.6 \%$ of the 31 loci in seven families, and in CTCT, $61.5 \%$ of the 13 loci in four families showed the less informative segregation patterns (types B, C and D). Five different loci showed segregation distortion: ADL0188 and ADL0192 (in family 4) and LEI0169 (in families 1, 6 and 7) in TCTC, and MCW0058 (in family 3), LEI0079 (in families 1 and 3), in addition to LEI0169 (in family 1) in CTCT (Table 1).

Lengths of linkage maps were 425.1, 231.6 and $433.1 \mathrm{cM}$ for TCTC, CTCT and the consensus, respectively (Figure 1A). The map was constructed in two segments only for CTCT (80.7 and $150.9 \mathrm{cM}$ ). Distances between two adjacent loci ranged from 0.6 to $54.8 \mathrm{cM}$ in TCTC (13.7 cM on average), from 1.1 to $58.3 \mathrm{cM}$ in CTCT (17.8 cM on average), and from 0.6 to $54.8 \mathrm{cM}$ in the consensus ( $13.5 \mathrm{cM}$ on average). No position inversions were observed, comparing the map of each population with the consensus map, except between MCW0145 and LEI0079 in the CTCT map, relative to the consensus.

Locus MCW0020 in the TCTC map and ADL0234, MCW0297, LEI0174, MCW0112, ADL0150, and LEIO079 in the CTCT map fell outside the confidence intervals of distances between loci, indicating significant position shifts relative to the consensus map (Figure 1A). With respect to the estimation of frequency distributions of locus orders (Figure 2A), the points concen- 


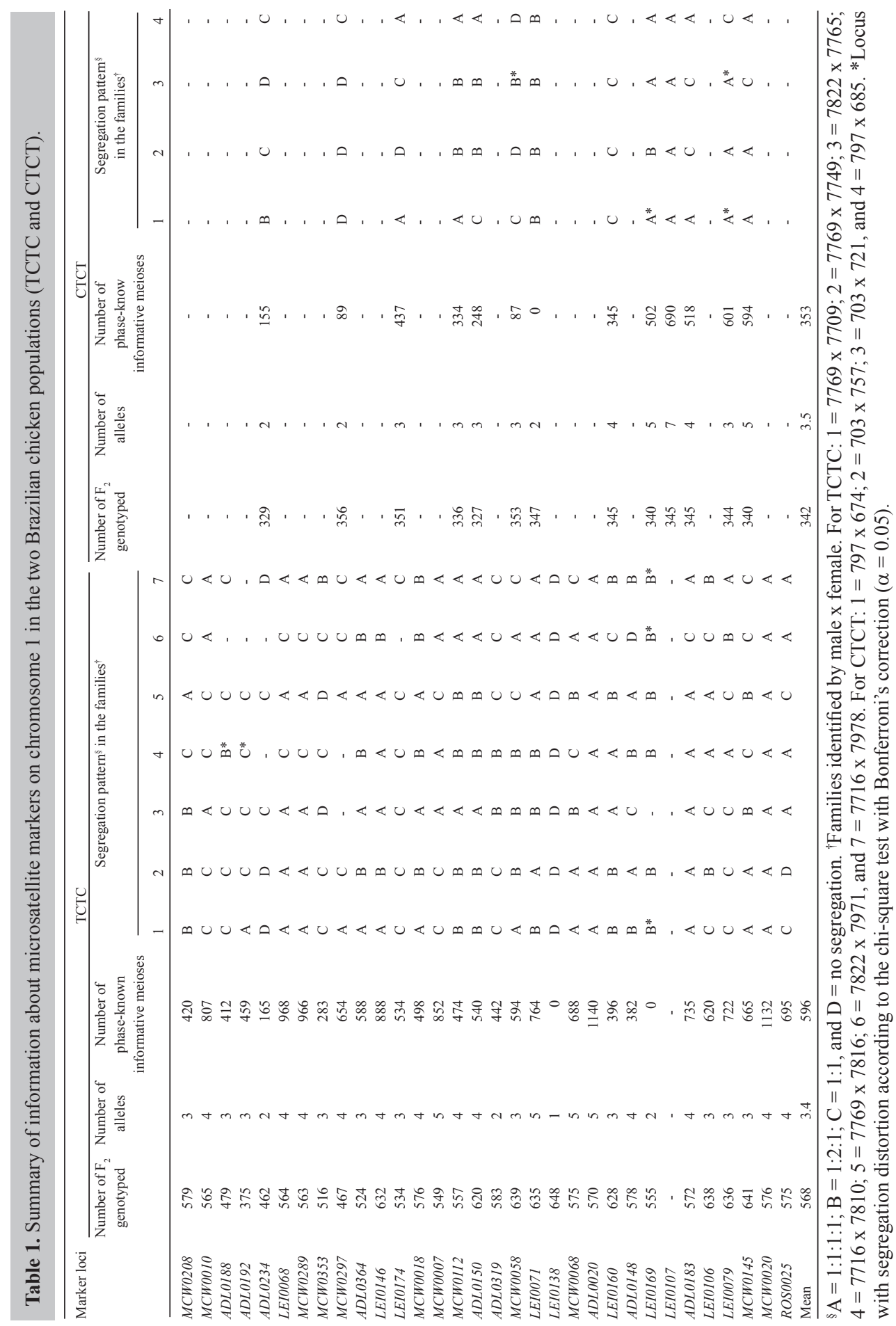


A

EMBRAPA - TCTC

EMBRAPA - TCTC/CTCT

EMBRAPA - CTCT
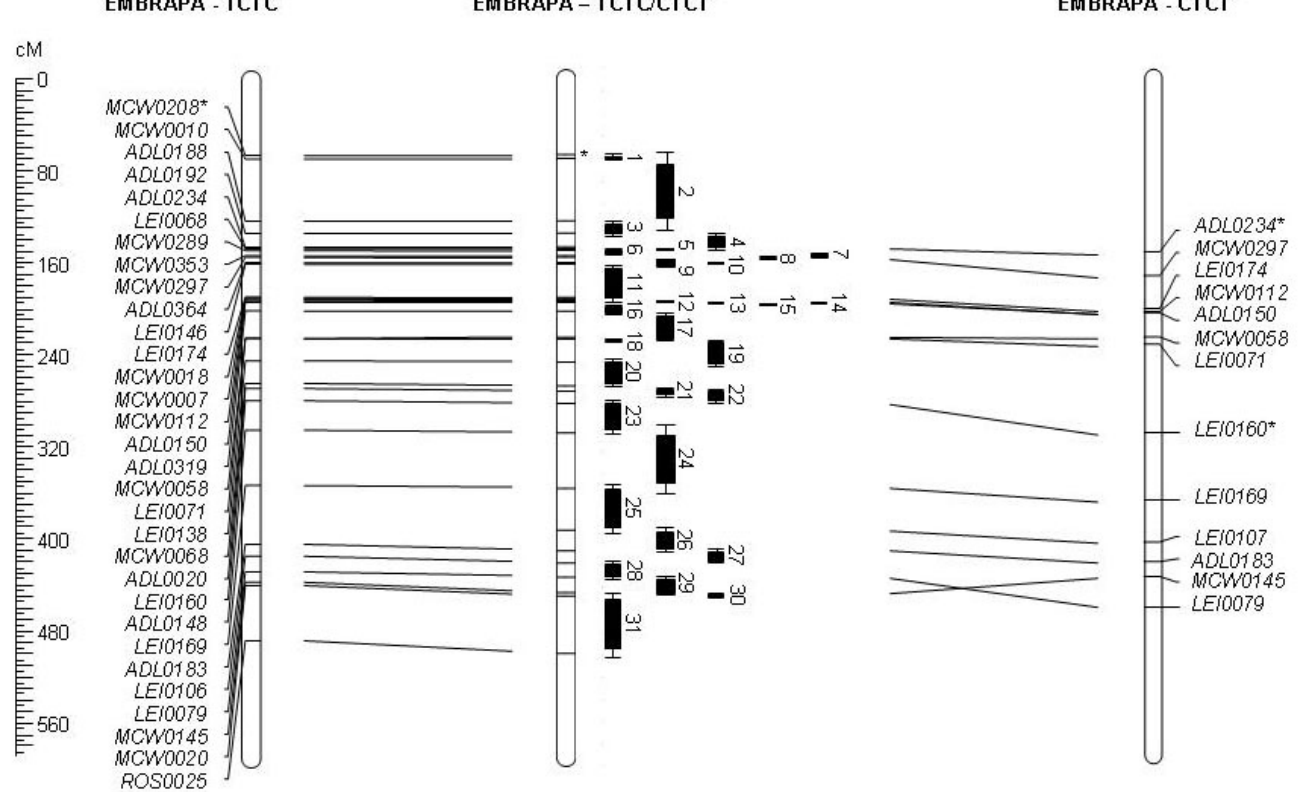

B

EMBRAPA - TCTC

EMBRAPA - TCTC/CTCT

EMBRAPA - CTCT
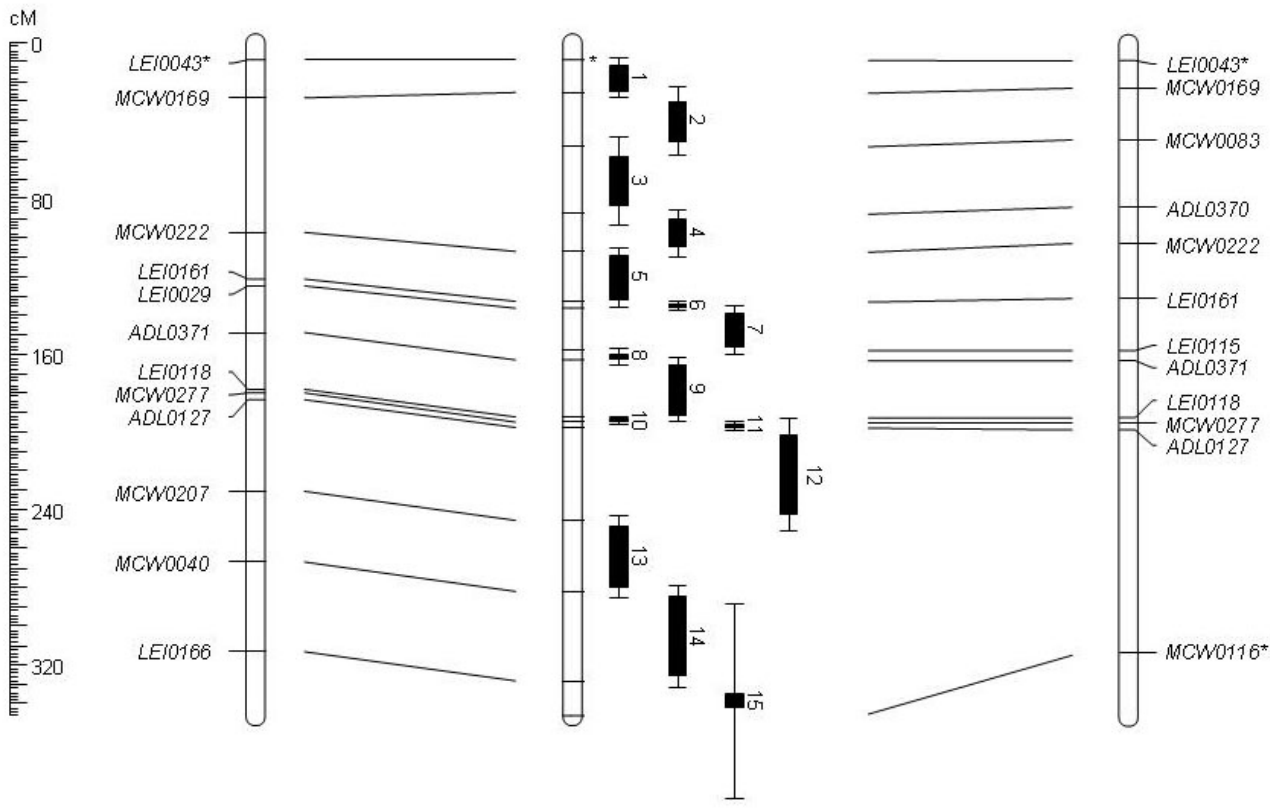

Figure 1. Continued on next page. 


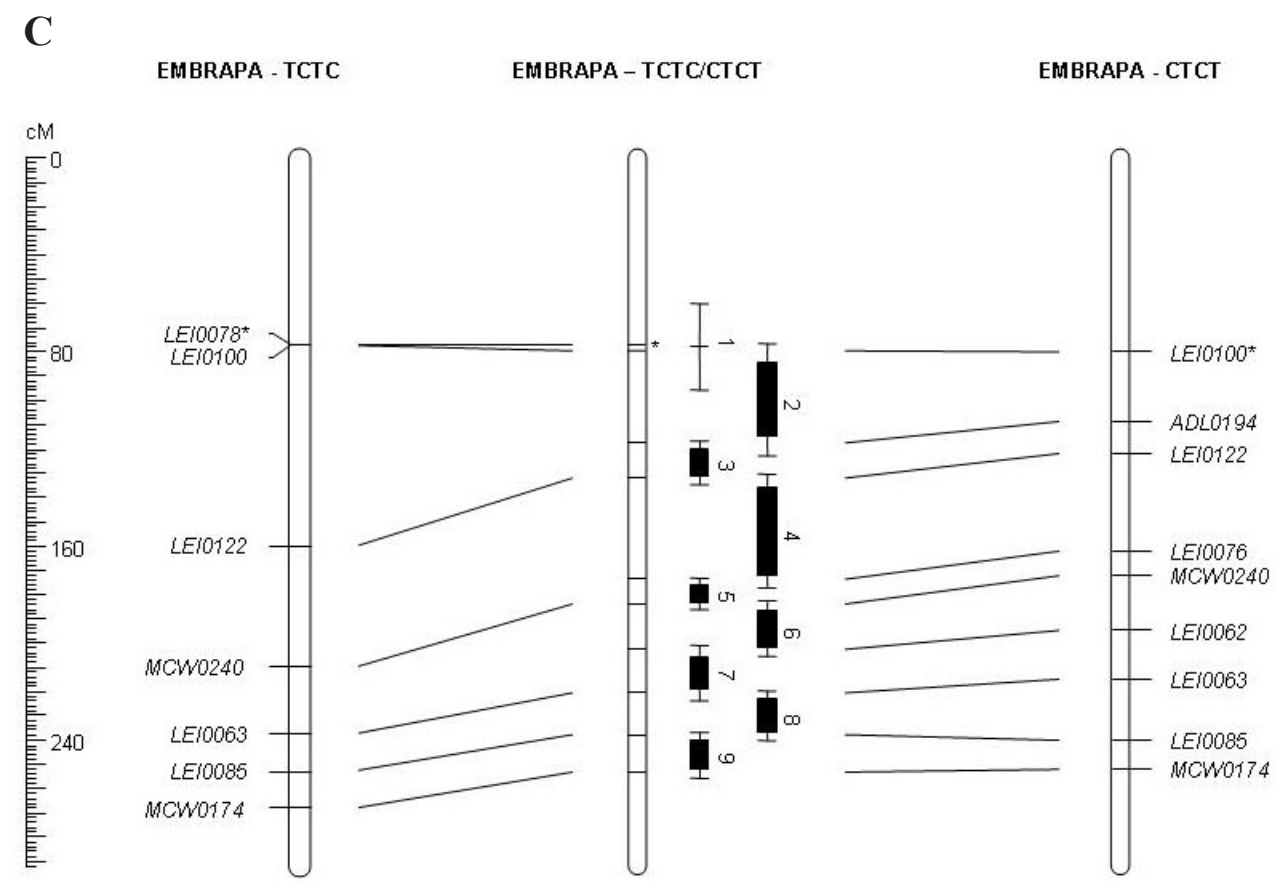

Figure 1. Representation of the single and consensus linkage maps constructed from two Brazilian reciprocal chicken populations. Each distance was numbered in the EMBRAPA - TCTC/CTCT consensus linkage map and has a confidence interval represented by boxes (lower limit) and bars (upper limit), using bootstrap sampling. *Marker position defined on the chicken consensus linkage map (Schmid et al., 2005 and ArkDB, http://www.thearkdb.org/). A. Chromosome 1 (GGA1). B. Chromosome 3 (GGA3). C. Chromosome 4 (GGA4).

trated on and around the diagonal, demonstrating that the orders formerly proposed for the consensus map were partially confirmed. When the intersection of orders between the axes "markers" and "positions" were considered in the bootstrap sampling, 22 loci did not exceed the $10 \%$ threshold, that is, in less than 100 samples no correspondence was found between these two axes. These loci were located between ADLO188 and LEI0107, except for MCW0289 and LEIO146. Another concentration of points outside of the diagonal was also observed, approximately between $M C W 0208$ and LEI0169 (loci 0 to 25 in the axis "markers"), revealing a possible inversion between two linkage groups: one between MCW0208 and LEI0169, which could also be in the opposite direction and another between LEIO107 and ROSOO25. This could be due to the fact that the CTCT map was built in two parts and there was a large gap between the two linkage groups: one from $A D L 0234$ to LEI0071 and another from LEI0160 to MCW0145. Although it was possible to use the information originated from both populations in the construction of the consensus map, a higher density of markers between LEI0071 and LEI0160 in CTCT could have prevented this inversion.

The greater similarity between TCTC and the consensus maps, than between CTCT and the consensus (Figure 1A), could be attributed to the higher number of phase-known informative meioses a posteriori, as well as of markers in TCTC, considering that only 13 loci were genotyped in CTCT, whereas 31 were genotyped in TCTC. Thirty-two loci were used in the consensus map. Whereas only 12 loci were simultaneously positioned in the TCTC and CTCT 
maps, 19 loci were genotyped exclusively in TCTC and one exclusively in CTCT (LEI0107). The frequency of type A segregation pattern (the most informative) was similar for TCTC $(39.4 \%)$ and CTCT $(38.5 \%)$. This seemed to be a critical factor for the estimation of confidence intervals of distances between adjacent loci, but not for determining locus positions.

The three largest confidence intervals (represented by bars) were found for distances 2,24 and 31 ( $60.9 \mathrm{cM}$ on average) and the three smallest (represented by boxes) were reported for distances 8,10 and $13(0.1 \mathrm{cM}$ on average) (Figure 1A). It should be noted that markers flanking distances $2,10,13$, and 31 were restricted to TCTC, whereas for the distances 8 and 24, MCW0297 and LEIO169 were genotyped in both populations. This indicates that the length of confidence intervals of distances depended on distances themselves, on the informativeness and segregation patterns of the flanking loci within and across families.

\section{$G G A 3$}

For the TCTC and CTCT populations, on average, 490 and $344 \mathrm{~F}_{2}$ individuals were genotyped, respectively, showing 3.2 and 3.1 alleles per locus and 517 and 351 phase-known informative meioses a posteriori (Table 2). However, $58.8 \%$ of loci in TCTC and $76.6 \%$ of loci in CTCT had the least informative segregation patterns (types B, C and D). Overall, five loci showed segregation distortion: ADL0127 (in family 5) in TCTC and LEI0043 and MCW0169 (both in family 2), $A D L 0370$ (in family 1) and MCW0222 (in families 2 and 3) in CTCT.

Figure 1B shows the maps, whose total lengths were $304.1,305.0$ and $336.7 \mathrm{cM}$ for TCTC, $\mathrm{CTCT}$ and the consensus, respectively. Distances between two adjacent loci ranged from 1.7 to $69.2 \mathrm{cM}$ in the TCTC map (25.3 cM on average), from 2.9 to $115.8 \mathrm{cM}$ in the CTCT map (25.4 $\mathrm{cM}$ on average) and from 2.3 to $47.6 \mathrm{cM}$ in the consensus map (21.1 cM on average). No position inversions were detected in the comparison between each population map and the consensus map.

Loci LEI0029, ADL0371, MCW0277, and ADL0127 in the TCTC map fell outside the confidence intervals of distances between loci, indicating significant position shifts relative to the consensus map (Figure 1B). The CTCT map, on the other hand, according to the same criteria, was identical to the consensus map. In the estimation of frequency distributions of locus orders (Figure 2B), a reasonable concentration of points on and around the diagonal was observed, allowing us to conclude that the orders initially proposed for the consensus map were partially confirmed. Thirteen loci did not exceed the minimum threshold of $10 \%$ when we considered the intersection between the axes "markers" and "positions". These loci were located between $M C W 0083$ and $M C W 0116$, except for $M C W 0040$.

The high similarity between the CTCT and the consensus map could reflect the higher saturation of markers between LEI0043 and ADL0127, which was on average $17.2 \mathrm{cM}$ in CTCT, whereas in TCTC it was $19.3 \mathrm{cM}$, also considering that the same number of markers (12) was employed in both populations and that the consensus map was constructed based on 16 loci (Figure 1B). The eight loci were simultaneously positioned on the TCTC and CTCT maps; four others were genotyped exclusively in TCTC, and another four in CTCT. The frequency of type A segregation pattern was $41.2 \%$ in TCTC and $23.4 \%$ in CTCT.

The three largest confidence intervals (bars) were found for distances 3, 12 and 14 (52.0 cM on average), excluding distance 15, which contained MCW0116 in TCTC, and the three shortest (boxes) for distances 6, 10 and 11 (1.6 cM on average) in CTCT (Figure 1B). Markers flanking distance 14 were limited to TCTC (MCW0040 and LEI0166), whereas for distance 3, only CTCT was genotyped with markers MCW0083 and ADL0370 (Figure 1B). 
M.F. Rosário et al.

1366

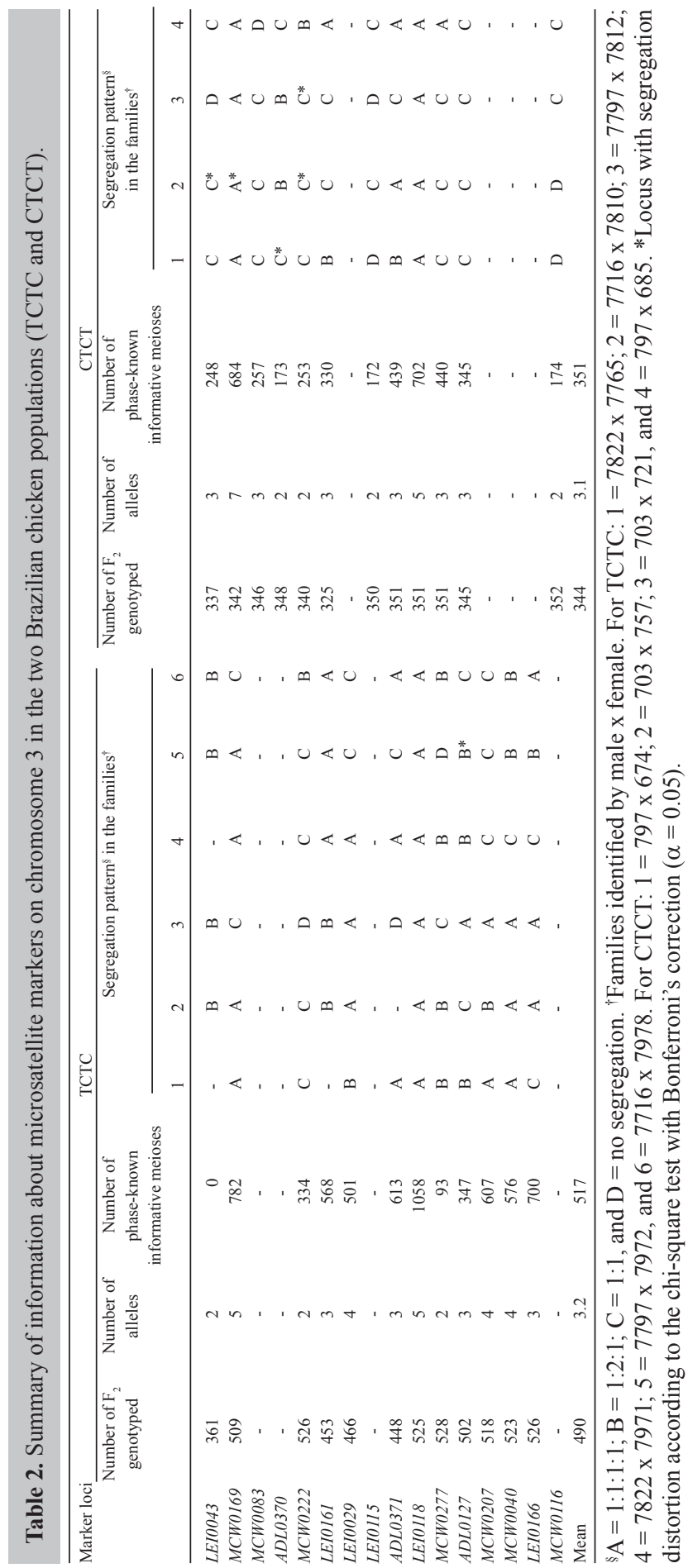

Genetics and Molecular Research 9 (3): 1357-1376 (2010)

CFUNPEC-RP www.funpecrp.com.br 
$G G A 4$

On average, $521 \mathrm{~F}_{2}$ chickens were genotyped in the TCTC population and 343 in the CTCT population, which presented 4.0 and 3.6 alleles per locus and 529 and 371 phase-known informative meioses a posteriori, respectively (Table 3 ). It was noticed that $68.3 \%$ of loci in TCTC and $63.9 \%$ in CTCT belonged to the less informative segregation patterns (types B, C and D). Overall, four different loci showed segregation distortion: LEIO100 and LEI0085 (both in family 4) and MCW0174 (in family 2) in TCTC and ADL0194 (also in family 4) in CTCT.

Total lengths of linkage maps were: $191.0,172.3$ and $176.4 \mathrm{cM}$ for TCTC, CTCT and the consensus, respectively (Figure 1C). Distances between two adjacent loci ranged from 0.3 to $82.5 \mathrm{cM}$ in TCTC (27.2 cM on average), from 10.0 to $40.4 \mathrm{cM}$ in CTCT $(19.1 \mathrm{cM}$ on average) and from 2.6 to $41.7 \mathrm{cM}$ in the consensus (17.6 cM on average). Similar to what was reported for $G G A 1$ and $G G A 3$, no position inversions were detected in the comparison between each population map and the consensus map.

Loci MCW0240 and MCW0174 in TCTC and MCW0240 in CTCT fell outside the confidence intervals of distances between adjacent loci, suggesting significant position shifts relative to the consensus map (Figure 1C). The estimation of frequency distributions of locus orders (Figure 2C) indicated that the points were scattered around the diagonal, showing that the orders initially proposed for the consensus map had low precision. Yet, six loci did not exceed the minimum threshold of $10 \%$ when we considered the intersection between the axes "markers" and "positions".

\section{A}

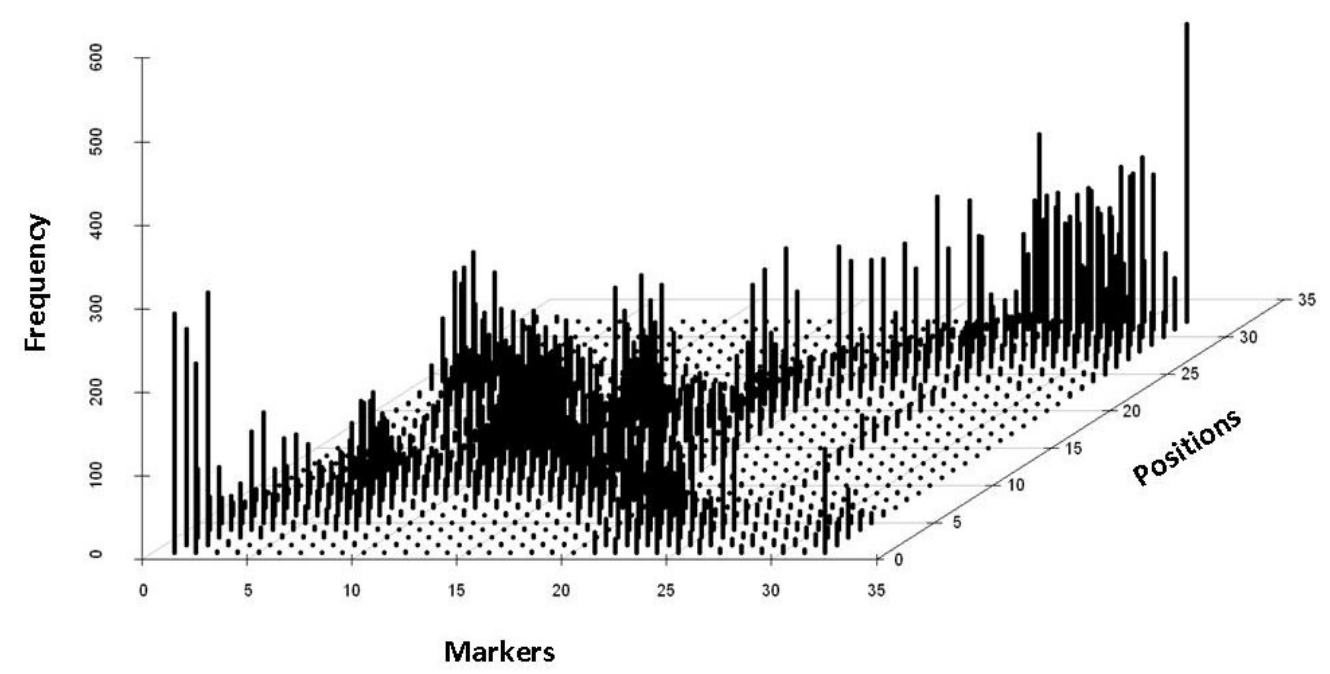

Figure 2. Continued on next page. 


\section{B}

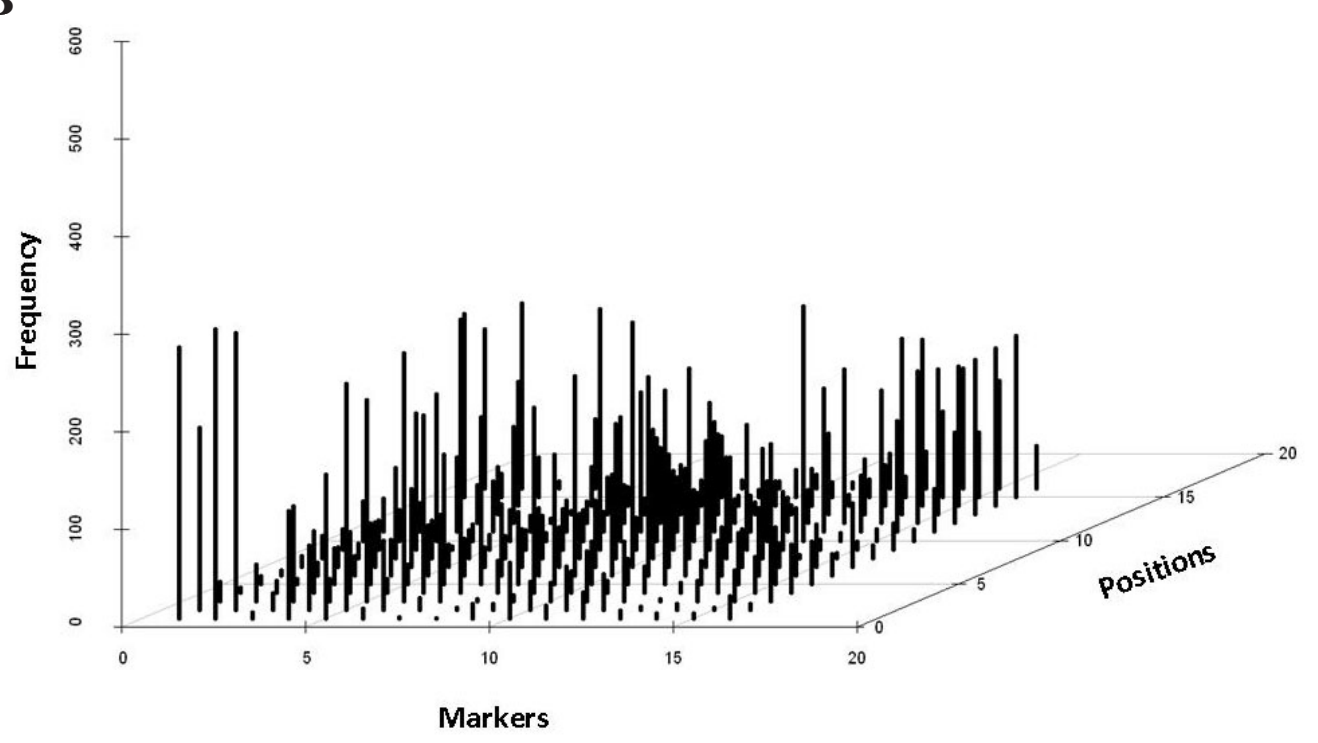

C

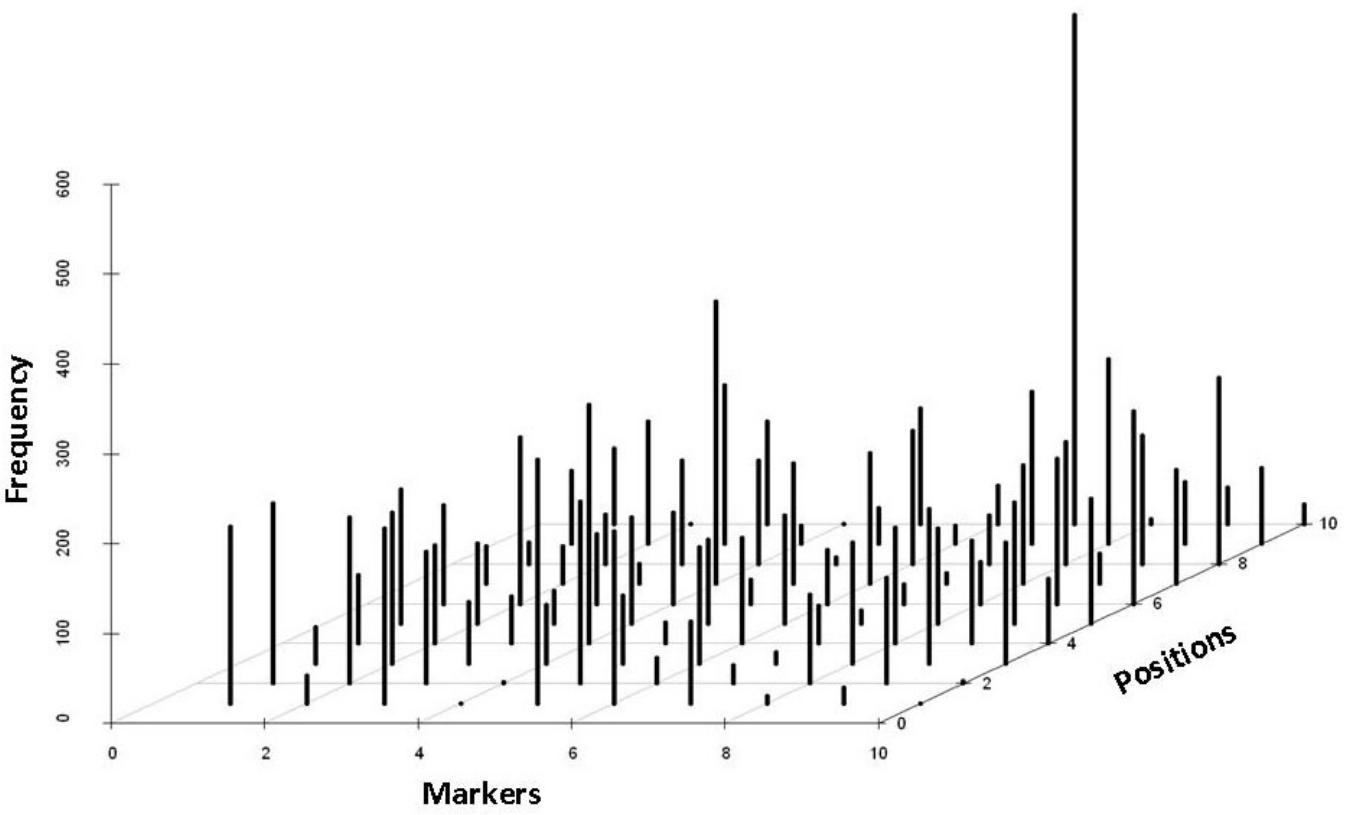

Figure 2. Distribution of frequencies between markers and positions using a seriation method. X-axis is the position of the markers on initial linkage map, y-axis is the frequency on the bootstrap sampling and z-axis is the locus position at each sample. A. Chromosome 1 (GGA1). B. Chromosome 3 (GGA3). C. Chromosome 4 (GGA4). 
Precision of microsatellite markers in chicken linkage maps

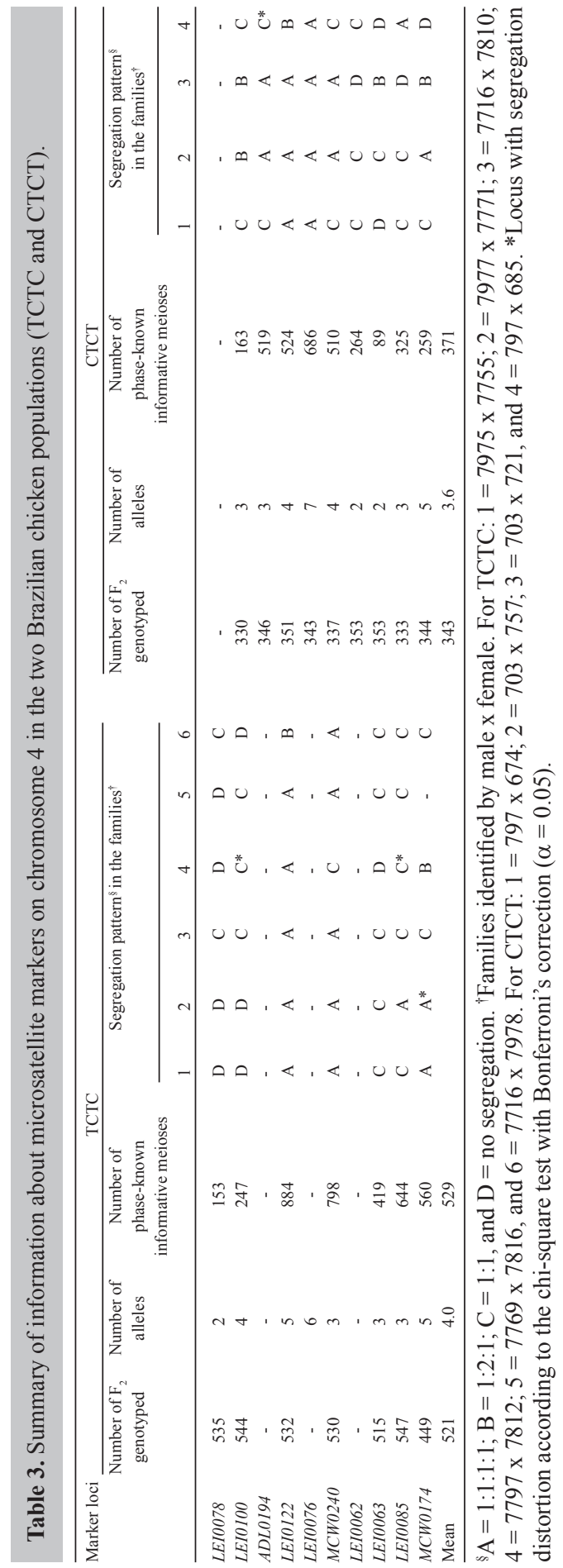


The CTCT map was more similar to the consensus than the TCTC map (Figure 1C). Overall, 10 loci were used in the construction of the consensus map; the TCTC population was genotyped with seven markers and the CTCT with nine. Six loci were used to genotype both TCTC and CTCT, one exclusively TCTC (LEI0078) and three CTCT (ADL0194, LEI0076 and LEI0062). The frequency of the type A segregation pattern was $43.3 \%$ in TCTC and $23.4 \%$ in CTCT.

The three largest confidence intervals (bars) were found for distances 1, 2 and 4 (43.0 $\mathrm{cM}$ on average) and the three smallest (boxes) for distances 1,3 and 5 (6.3 cM on average) (Figure 1C). Both populations were genotyped with markers flanking all these distances, except for LEIO078, restricted to TCTC and ADL0194 and LEI0076, to CTCT.

\section{Brazilian, International and genomic consensus maps}

The Brazilian Consensus Map (BCM) was assumed to be a satisfactory estimate for GGA1, GGA3 and GGA4. Consequently, comparisons of BCM with the International Consensus Map (ICM) reported by Schmid et al. (2005), and also with the loci positions described by UniSTS (http://www.ncbi.nlm.nih.gov/unists) on the chicken genome sequence (GS) (Hillier et al., 2004) were carried out. In order to compare these three maps, 1:0.341, 1:0.329 and 1:0.321 cM:Mb relationships were adopted for GGA1, GGA3 and GGA4, respectively, according to the ArkDB (http://www.thearkdb.org) and Schmid et al. (2005), NCBI Map Viewer (http://www.ncbi.nlm.nih.gov/mapview) and Hillier et al. (2004). In general, the three chromosomes showed variable distances and ordering of microsatellite markers (Figure 3A, B and C).

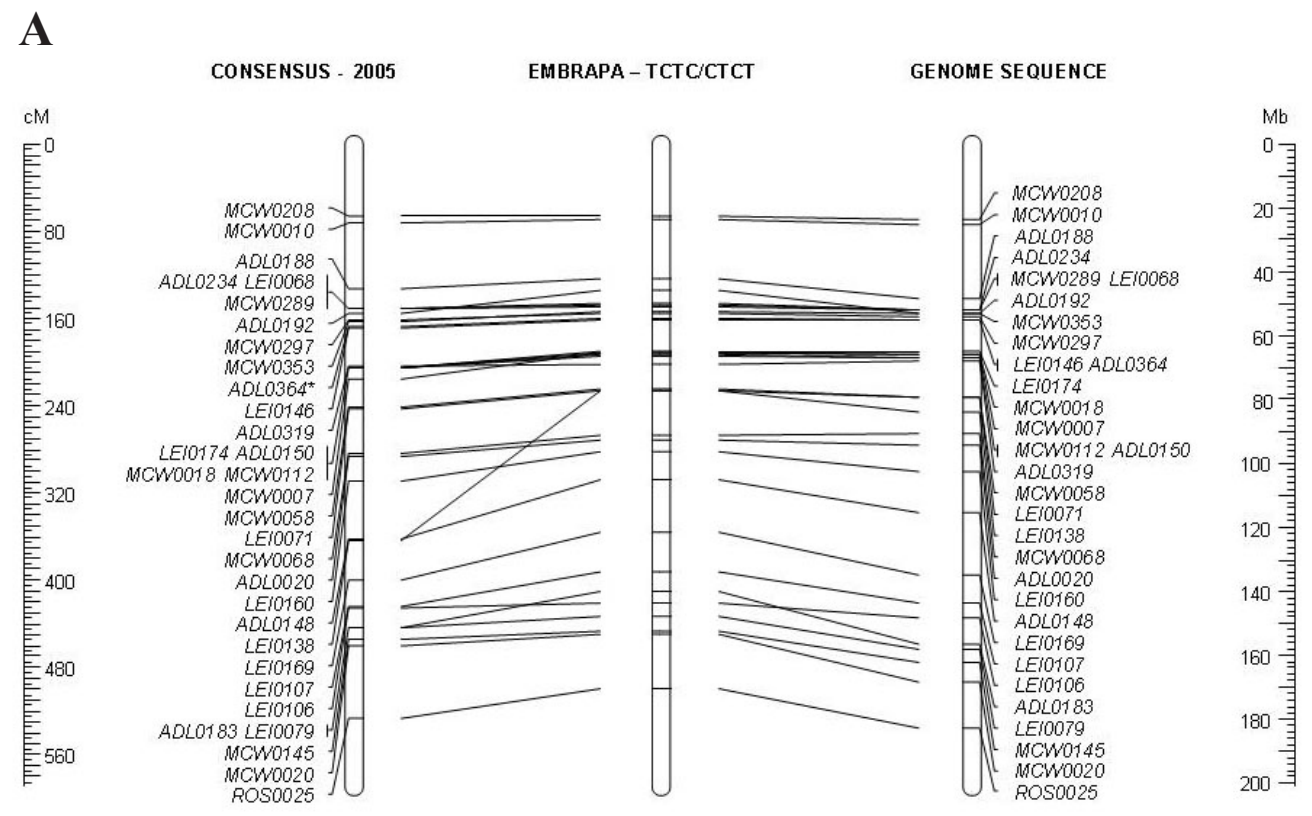

Figure 3. Continued on next page. 
B

CONSENSUS - 2005

EMBRAPA - TCTC/CTCT

GENOME SEQUENCE
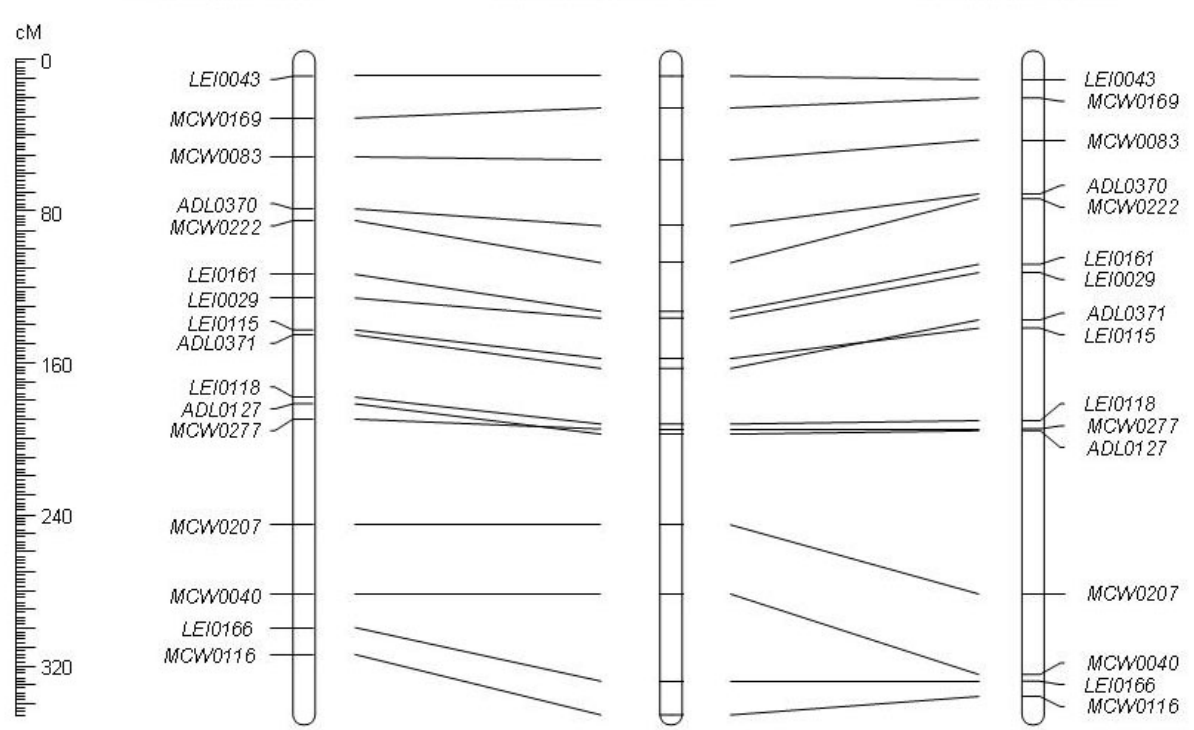

20 単

C
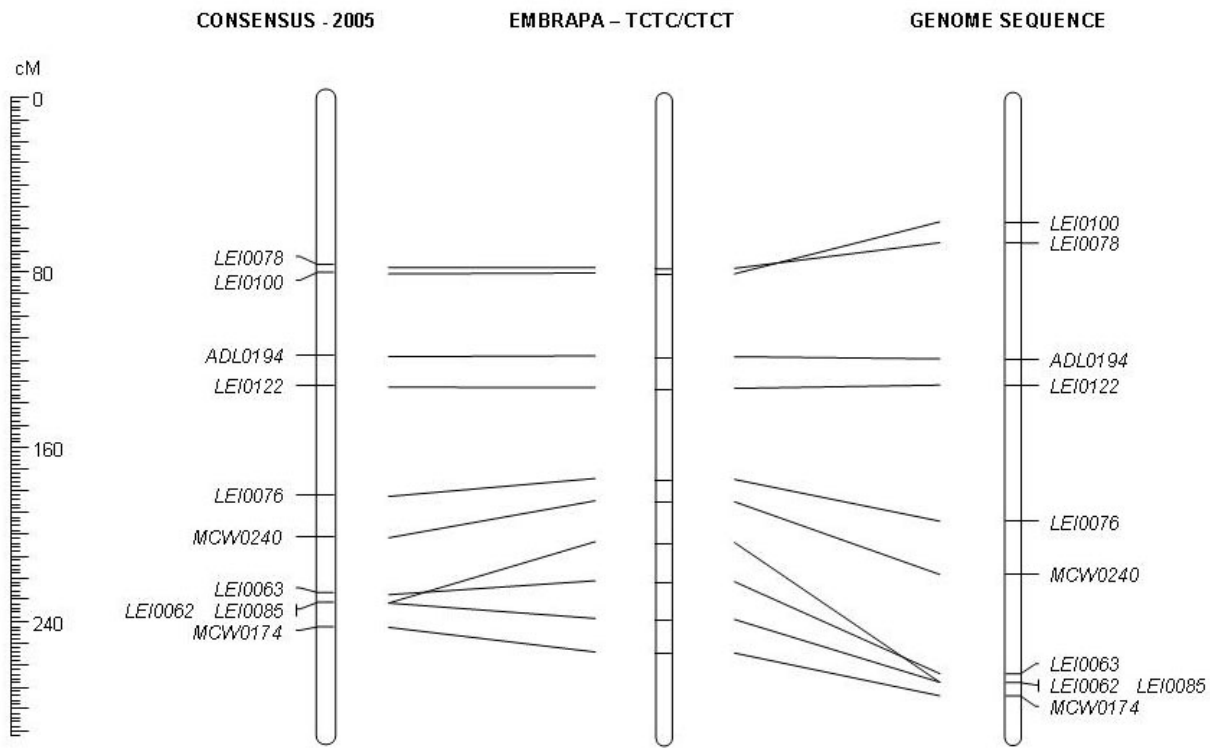

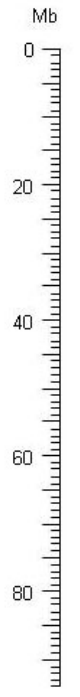

Figure 3. Comparison between Brazilian chicken consensus (center) and chicken consensus linkage maps (Schmid et al., 2005 and ArkDB, http://www.thearkdb.org/) (left) both in cM (left scale) and the marker position according to UniSTS (http://www.ncbi.nlm.nih.gov/unists) on the chicken genome sequence (Hillier et al., 2004 and NCBI/ MapViewer, http://www.ncbi.nlm.nih.gov/projects/mapview/) (right) in Mb (right scale). A. Chromosome 1 (GGA1); *Marker position defined according to Jennen et al. (2005). B. Chromosome 3 (GGA3). C. Chromosome 4 (GGA4). 
For $G G A 1,19$ position inversions were observed in the comparison between BCM and ICM and seven between BCM and GS. Locus LEI0138 showed a marked position shift in BCM compared to ICM; its position in GS, however, was similar to BCM. This result reinforced the hypothesis that $\mathrm{BCM}$ is a satisfactory estimate for GGA1. Twenty-one loci (65.0\%) fell outside the confidence intervals of distances in the comparison between BCM and ICM, and $13(40.6 \%)$ between BCM and GS, which denotes a significant position change.

For $G G A 3$, one inversion was observed between BCM and ICM and another between BCM and GS. Four of 16 loci fell outside the confidence intervals of distances in the comparison between $\mathrm{BCM}$ and ICM, and three of 16 between BCM and GS, which denotes significant position change.

For GGA4, one inversion was observed between BCM and ICM and two between $\mathrm{BCM}$ and GS. A single locus of 10 fell outside the confidence intervals of distances in the comparison between BCM and ICM, and seven of 10 loci between BCM and GS; the latter denotes a significant position change.

\section{DISCUSSION}

Our study was motivated by the difficulty in comparing genetic maps from different chicken populations. Confidence intervals for distances and frequency distributions for orders of microsatellite markers were estimated. Confidence intervals for SNP positions had been already calculated for humans (Matise et al., 2007) and bovines (Snelling et al., 2005). This approach can be used to obtain more precise and detailed maps, which are essential for QTL mapping and gene searches. These are the first genetic maps constructed based on reciprocal crosses between broiler and layer chicken lines generated under Brazilian climate, nutrition and management conditions.

Genetic maps for GGA1, GGA3 and GGA4 were estimated separately for each of the two Brazilian populations developed for mapping QTLs associated with growth performance and carcass traits. Consensus maps including the genotypes from both populations were also estimated, totaling 475, 434 and 450 phase-known informative meioses a posteriori for GGA1, $G G A 3$ and $G G A 4$, respectively (average of the two populations). These numbers are higher than those reported by Schmid et al. (2000) for the East Lansing and Compton populations, but lower than those presented for the Wageningen population (with average mapping resolution of $1 \mathrm{cM}$ ). These three populations were used in the construction of the International Chicken Consensus Map by Groenen et al. (2000), which was afterwards updated by Schmid et al. (2000, 2005).

Therefore, the consensus maps presented here give satisfactory estimates of the positions and orders of the microsatellite loci in this species. Due to the fact that the domestic chicken (Gallus gallus domesticus) originated from a single species (the Red Jungle Fowl - Gallus gallus), differences in estimates of locus positions and orders, and of map lengths, between different studies could be explained by their distinct genetic background, the experimental population design, population size, and type, number of and distance between molecular markers, number of phase-known informative meioses, genotyping errors, locus segregation patterns, number of lost genotypes and sex, besides the several statistical methods employed in the analyses (Hackett and Broadfoot, 2003). Another point is that the chicken genome sequence was obtained from a single Red Jungle Fowl female (Hillier et al., 2004), which could result in unrealistic differences due to a sampling error. Consequently, several factors complicate the comparisons among genetic maps originated from different studies. 
Based on the estimated confidence intervals, there were some discrepancies both in distances between adjacent loci and in orders of loci, which in many cases could be attributed to chance. Others could be due to reciprocal crossing, to the sampling error of the TCTC and CTCT founder animals, or to the number of phase-known informative meioses a posteriori. Additionally, the genetic background of these two populations were the White Cornish, White Plymouth Rock and New Hampshire breeds for the broiler line (TT) and the White Leghorn breed for the layer line (CC), whereas for the populations used in the construction of the International Consensus Map (Groenen et al., 2000), the genetic background was Jungle Fowl and White Leghorn for East Lansing, White Leghorn for Compton and White Plymouth Rock for the Wageningen population. This could also in part explain the differences observed.

The estimated confidence intervals and frequency distributions helped measure the precision of locus distances and orders, respectively, and facilitated the comparisons of GGA1, GGA3 and GGA4 Brazilian Consensus Maps with estimates from independent studies. According to Matise et al. (2007), these maps represent resampling trials, therefore bias associated with locus position and order estimates can be detected. This is important because, in general, the uncertainty effects associated with the construction of linkage maps are ignored. Also, the detection of genotyping errors and the haplotype interference can be negatively affected by the uncertainty of the linkage map. Other studies have also demonstrated that incorrect estimates of distances between loci negatively affect multipoint linkage analyses (Halpern and Whittemore, 1999; Daw et al., 2000).

For the TCTC population, Nones et al. (2005) had already constructed a $464.1 \mathrm{cM}$ linkage map for GGA1, using 26 microsatellite markers, with an average distance between adjacent loci of $15.0 \mathrm{cM}$. In our study, the TCTC map for GGAl was reanalyzed using 31 microsatellite markers and showed a total length of $491.1 \mathrm{cM}$, with $13.7 \mathrm{cM}$ average distance between adjacent loci. Two inversions were observed, comparing the results shown here with those from Nones et al. (2005): between MCW0208 and MCW0010 and between ADL0183 and LEI0106. However, those authors did not employ the $A L L$ function in the CRI-MAP software; they used the TWOPOINT, BUILD, FLIPS2, and CHROMPIC functions, which could be an explanation for the differences.

We noted that the Brazilian consensus map for $G G A 1$ was more similar to the genome sequence than the International Consensus Map. Locus LEI0138 showed a position discrepancy in the International Consensus Map, which has been extensively used by researchers for QTL mapping in chickens. A search for QTLs flanked by this locus in the Chicken QTL Database (http://www.animalgenome.org/QTLdb/chicken.html), revealed that only Nones et al. (2006) mapped QTLs for body weight at 35 days of age (in the linecross analysis) and for liver weight adjusted for body weight at 42 days of age (in the half-sib analysis) using the TCTC population. Therefore, it is suggested that the position of this locus be revised in order to allow new QTLs to be mapped.

Although the CRI-MAP software (Green et al., 1990) has been widely used by the scientific community, it does not include an option for confidence interval estimation. Therefore, it is not possible to determine the precision of multipoint estimates of locus distances and orders. However, due to the fact that the program was written in LINUX, it was possible to make a few changes and the bootstrap sampling in association with the R software to estimate such intervals. Another limitation of CRI-MAP is that it provides biased estimates when there is missing data (Stewart and Thompson, 2006). Averaging across loci, 14.1, 13.0 and 12.2\% 
genotypes were missing for $G G A 1, G G A 3$ and $G G A 4$, respectively, in our present study.

Employing simulations, Hackett and Broadfoot (2003) noted that missing data and/ or genotyping errors reduced the proportion of correctly ordered maps. The problem was even worse when loci were close to each other. These authors also reported that missing data resulted in shorter maps when distances between markers were larger. As a result of the missing data, information about the true number of recombinations that occurred along the chromosome was lost. Buetow (1991), also using simulations, demonstrated that $1 \%$ undetected genotyping errors leads to incorrect ordering of loci, lengthening the map, particularly when the density of loci is increased. In our study, missing genotypes did not seriously compromise locus order estimates, but it may have influenced the estimation of distances between loci.

Here, 14 loci presented segregation distortion according to established patterns (Tables 1, 2 and 3), but this did not influence the construction of maps, similar to what was found by Hackett and Broadfoot (2003). Xu (2008) has shown that the power to detect QTLs may benefit from segregation distortion for QTLs with additive effects. This author found that if there were loci with segregation distortions that were ignored in QTL mapping, there was a slight loss in power; but it was insignificant if the genome was densely covered by markers.

Along this line, the density of chicken linkage maps has been increased by using SNP markers, mining from the chicken genome sequence, as well as by developing high throughput genotyping platforms, such as the 60K SNP Illumina iSelect chicken array developed by the USDA Chicken GWMAS Consortium, Cobb Vantress and Hendrix Genetics. The map obtained by Groenen et al. (2009), containing 8599 SNPs, was a great advance in this direction and suggested a functional relationship between recombination frequency and GC-rich cohesin binding sites. Thus, SNPs could be useful to clarify uncertainties about positions and orders of loci and could complement the results from our study, since variations in distances and orders of microsatellite loci were found. Additionally, the integration of accurate genetic maps, BAC-based physical maps, radiation hybridization, and SNPs, would allow complete coverage of the chicken genome. The study of Aerts et al. (2003) and Ren et al. (2003) improved the alignment of physical and linkage maps by providing a large number of BACs and molecular markers. However, Wallis et al. (2004) reported that BACs covered only 95\% of the chicken genome and that the remaining 5\% should be covered by SNPs.

Finally, the chicken genetic linkage map of chromosomes 1, 3 and 4 with precision measures of microsatellite locus distances and orders, using bootstrap sampling allowed us to account for part of the discrepancies found among the different studies. The extension of these analyses to the remaining chromosomes could contribute to the comparison and integration of several genetic maps, allowing map construction and QTL mapping meta-analyses. In this way, QTLs could be mapped with increased precision in the estimates of positions and effects.

\section{ACKNOWLEDGMENTS}

We are grateful to EMBRAPA/PRODETAB and FAPESP for financial support and to K. Nones, E.E. Baron and D.C. Ruy for generation of the genotypic data. M.F. Rosário received scholarships from FAPESP (\#04/02080-2) and CNPq (\#152500-2007/3), G.R.A. Margarido and C. Boschiero received scholarships from FAPESP. A.S.A.M.T. Moura, L.L. Coutinho and A.A.F. Garcia are recipients of research productivity scholarships from CNPq. 


\section{REFERENCES}

Abasht B, Dekkers JC and Lamont SJ (2006). Review of quantitative trait loci identified in the chicken. Poult. Sci. 85: 2079-2096.

Aerts J, Crooijmans R, Cornelissen S, Hemmatian K, et al. (2003). Integration of chicken genomic resources to enable whole-genome sequencing. Cytogenet. Genome Res. 102: 297-303.

Ambo M, Campos RLR, Moura ASAMT, Boschiero C, et al. (2008). Genetic linkage maps of chicken chromosomes 6, 7, 8, 11 and 13 from a Brazilian resource population. Sci. Agric. 65: 447-452.

Ambo M, Moura AS, Ledur MC, Pinto LF, et al. (2009). Quantitative trait loci for performance traits in a broiler $\mathrm{x}$ layer cross. Anim. Genet. 40: 200-208.

Buetow KH (1991). Influence of aberrant observations on high-resolution linkage analysis outcomes. Am. J. Hum. Genet. 49: 985-994.

Buetow KH and Chakravarti A (1987). Multipoint gene mapping using seriation. I. General methods. Am. J. Hum. Genet. 41: 180-188.

Bumstead N and Palyga J (1992). A preliminary linkage map of the chicken genome. Genomics 13: 690-697.

Campos RL, Nones K, Ledur MC, Moura AS, et al. (2009). Quantitative trait loci associated with fatness in a broiler-layer cross. Anim. Genet. 40: 729-736.

Darvasi A and Soller M (1992). Selective genotyping for determination of linkage between a marker locus and a quantitative trait locus. Theor. Appl. Genet. 85: 353-359.

Daw EW, Thompson EA and Wijsman EM (2000). Bias in multipoint linkage analysis arising from map misspecification. Genet. Epidemiol. 19: 366-380.

Efron B and Tibshirani RJ (1993). An Introduction to the Bootstrap. 1st edn. Chapman and Hall/CRC, New York.

Figueiredo EAP, Rosa PS, Scheuermann GN, Jaenisch FRF, et al. (2003a). Genetic gain in body weight feed conversion and carcass traits in White Plymouth Rock broiler strain Embrapa 021. Proceedings of the IX World Conference on Animal Production, Porto Alegre.

Figueiredo EAP, Schmidt GS, Ledur MC and Avila VS (2003b). Genetic gain in egg production and egg weight in White Legorn Embrapa 011. Proceedings of the IX World Conference on Animal Production, Porto Alegre.

Fishelson M and Geiger D (2002). Exact genetic linkage computations for general pedigrees. Bioinformatics 18 (Suppl 1): S189-S198.

Green P, Falls K and Crooks S (1990). Documentation for CRIMAP, version 2.4. Washington University School of Medicine, St. Louis. Available at [http://linkage.rockefeller.edu/soft/crimap/]. Accessed October 20, 2009.

Groenen MA, Crooijmans RP, Veenendaal A, Cheng HH, et al. (1998). A comprehensive microsatellite linkage map of the chicken genome. Genomics 49: 265-274.

Groenen MA, Cheng HH, Bumstead N, Benkel BF, et al. (2000). A consensus linkage map of the chicken genome. Genome Res. 10: 137-147.

Groenen MA, Wahlberg P, Foglio M, Cheng HH, et al. (2009). A high-density SNP-based linkage map of the chicken genome reveals sequence features correlated with recombination rate. Genome Res. 19: 510-519.

Hackett CA and Broadfoot LB (2003). Effects of genotyping errors, missing values and segregation distortion in molecular marker data on the construction of linkage maps. Heredity 90: 33-38.

Halpern J and Whittemore AS (1999). Multipoint linkage analysis. A cautionary note. Hum. Hered. 49: 194-196.

Hillier LW, Miller W, Birney E and Warren W (2004). Sequence and comparative analysis of the chicken genome provide unique perspectives on vertebrate evolution. Nature 432: 695-716.

Jennen DG, Vereijken AL, Bovenhuis H, Crooijmans RM, et al. (2005). Confirmation of quantitative trait loci affecting fatness in chickens. Genet. Sel. Evol. 37: 215-228.

Kosambi DD (1944). The estimation of map distance from recombination values. Ann. Eugenics 12: 172-175.

Levin I, Santangelo L, Cheng H, Crittenden LB, et al. (1994). An autosomal genetic linkage map of the chicken. J. Hered. 85: 79-85.

Liu BH (1998). Statistical Genomics: Linkage, Mapping and QTL Analysis. CRC Press, Boca Raton.

Matise TC, Chen F, Chen W, De La Vega FM, et al. (2007). A second-generation combined linkage physical map of the human genome. Genome Res. 17: 1783-1786.

Mollinari M, Margarido GR, Vencovsky R and Garcia AA (2009). Evaluation of algorithms used to order markers on genetic maps. Heredity 103: 494-502.

Nones K, Ledur MC, Ruy DC, Baron EE, et al. (2005). Genetic linkage map of chicken chromosome 1 from a Brazilian resource population. Sci. Agric. 62: 12-17.

Nones K, Ledur MC, Ruy DC, Baron EE, et al. (2006). Mapping QTLs on chicken chromosome 1 for performance and 
carcass traits in a broiler x layer cross. Anim. Genet. 37: 95-100.

Ren C, Lee MK, Yan B, Ding K, et al. (2003). A BAC-based physical map of the chicken genome. Genome Res. 13: 2754-2758.

Rosário MF, Ledur MC, Moura ASAMT, Coutinho LL, et al. (2009). Genotypic characterization of microsatellite markers in broiler and layer selected chicken lines and their reciprocal F1s. Sci. Agri. 66: 150-158.

Schmid M, Nanda I, Guttenbach M, Steinlein C, et al. (2000). First report on chicken genes and chromosomes 2000. Cytogenet. Cell Genet. 90: 169-218.

Schmid M, Nanda I, Hoehn H, Schartl M, et al. (2005). Second report on chicken genes and chromosomes 2005. Cytogenet. Genome Res. 109: 415-479.

Snelling WM, Casas E, Stone RT, Keele JW, et al. (2005). Linkage mapping bovine EST-based SNP. BMC Genomics 6: 74.

Stewart WC and Thompson EA (2006). Improving estimates of genetic maps: a maximum likelihood approach. Biometrics 62: 728-734.

Voorrips RE (2002). MapChart: software for the graphical presentation of linkage maps and QTLs. J. Hered. 93: 77-78.

Wallis JW, Aerts J, Groenen MA, Crooijmans RP, et al. (2004). A physical map of the chicken genome. Nature 432: 761-764.

Wang J, He X, Ruan J, Dai M, et al. (2005). ChickVD: a sequence variation database for the chicken genome. Nucleic Acids Res. 33: D438-D441.

Wong GK, Liu B, Wang J, Zhang Y, et al. (2004). A genetic variation map for chicken with 2.8 million single-nucleotide polymorphisms. Nature 432: 717-722.

Xu S (2008). Quantitative trait locus mapping can benefit from segregation distortion. Genetics 180: 2201-2208. 